\title{
CASO 2016: Lupus Eritematoso Sistémico con manifestaciones iniciales de una Enfermedad de Kikuchi-Fujimoto
}

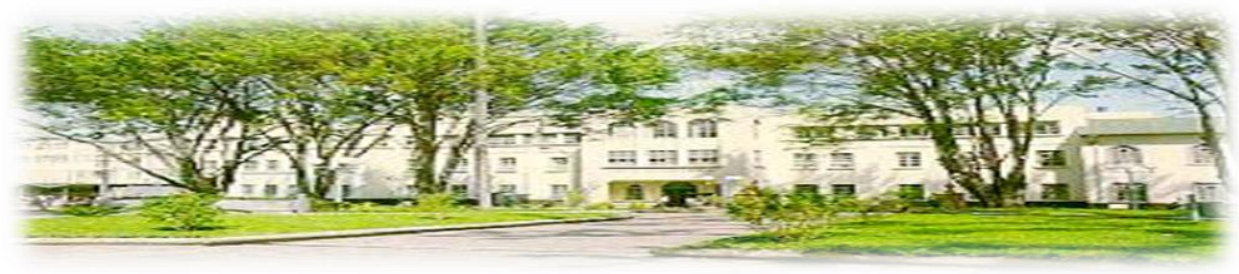

Hospital San Juan de Dios, San José, Costa Rica. Fundado en 1845

Recibido:

$05 / 12 / 2016$

Aceptado:

$16 / 12 / 2016$

Erick Molina Guevara ${ }^{1}$ Mónica Méndez Ceciliano ${ }^{2}$ Allan Umaña Herrera ${ }^{3}$

${ }^{1}$ Medico Asistente Especialista en Reumatología, Hospital México. Correo electrónico: elimolofra@ @otmail.com

${ }^{2}$ Medico Asistente Especialista en Reumatología, Hospital México. Correo electrónico: mon-

icamence@gmail.com

${ }^{3}$ Medico Asistente Especialista en Reumatología, Hospital México. Correo electrónico: aluma27@ hotmail.com

\section{INTRODUCCIÓN}

La enfermedad de Kikuchi-Fujimoto (EKF) es una enfermedad poco común, benigna y auto limitada, que puede verse ocasionalmente asociada con el Lupus Eritematoso Sistémico (LES), pudiendo presentarse antes, después o simultáneamente ${ }^{(1-5-6-8-13)}$. A continuación, se presentará y se analizará un caso clínico donde se demuestra la interrelación entre ambas patologías, este es un caso del Servicio de Reumatología del Hospital México, Costa Rica del año 2014.

\section{DESCRIPCIÓN DEL CASO CLÍNICO}

Femenina de 22 años de edad, conocida sana vecina del Valle Central, estudiante universitaria que ingresó al Centro medico para estudios por un síndrome febril que asociaba adenopatías cervicales número 2 ubicadas en el triángulo cervical posterior, gonalgía bilateral de 6 meses de evolución, pérdida de peso de 6 kilogramos y síntomas constitucionales.

$\mathrm{Al}$ examen físico se describen lesiones papulares oscuras en piel de cuero cabelludo, cara y tórax anterior, caída de cabello y ulceras orales. Durante 
su internamiento hay descripción de puños incompletos y artralgias, sin describir franca sinovitis.

Al momento del ingreso las pruebas de laboratorio evidenciaron linfopenia 800 totales, anemia normocítica normocrómica (hemoglobina $8.8 \mathrm{~g} / \mathrm{dl}$, hematocrito $30.2 \%$, volumen corpuscular medio $82 \mathrm{fl}$, hemoglobina corpuscular media $27 \mathrm{pg}$ ) y VSG 108 mm/h. En la bioquímica sanguínea, presento aumento de las globulinas $4,7 \mathrm{~g} / \mathrm{dl}$, con una albúmina de $3.5 \mathrm{~g} / \mathrm{dl}$, en el examen general de orina presentaba proteínas $25 \mathrm{mg} / \mathrm{dl}$ y en el sedimento no existían alteraciones. Se le realizo orina de 24 horas la cual reflejo $405 \mathrm{mg} / 24$ horas.

Las serologías analizadas fueron VIH, hepatitis B y $\mathrm{C}$, toxoplasma, rubéola, virus de Epstein-Barr y citomegalovirus, parvirus B19, las cuales fueron negativas; al igual que los urocultivos y hemocultivos realizados en diferentes momentos de su internamiento y la prueba de Mantoux.

Se le realizó una punción de médula ósea con aspirado, biopsia y cultivos que fueron negativos.

En las pruebas de autoinmunidad destacaba: ANA positivo a 3,2, C3 $82.7 \mathrm{mg} / \mathrm{dl}$ y C4 $10.7 \mathrm{mg} / \mathrm{dl}$ anti-ADN 60.4 UI/ml, factor reumatoide negativo.

En la TC toracoabdominal se objetivaron múltiples adenopatías de aspecto reactivo a nivel cervical y axilar y hepatomegalia. Ecocardiograma con una fracción de eyección del $60 \%$ dentro de límites considerados normales

Se le realizo biopsia de una adenopatía cervical donde se reporta ganglio linfático con preservación de la arquitectura linfoide e hiperplasia de folículos con centros germinales, presencia de macrófagos con cuerpos tingibles, y preservación de la zona del manto, se observan zonas de necrosis, correspondiente a una linfadenitis reactiva.

La biopsia de piel presenta epidermis conservada y edema de la dermis papilar, en la dermis reticular alta hay inflamación perivascular e intersticial, constituida principalmente por histiocitos de citoplasma claro granular y asociado a detritus nucleares. Cambios que sugieren enfermedad de kikuchi.

Se le inicio tratamiento con Prednisona $25 \mathrm{mg} / \mathrm{día}$ VO, Plaquinol $400 \mathrm{mg} /$ día VO y Azatioprina 50 $\mathrm{mg} /$ día VO, lo que conlleva a cese de la fiebre, disminución del tamaño de las adenopatías, elevación de la hemoglobina a cifras mayores de 12 $\mathrm{g} / \mathrm{dl}$, corrección de la proteinuria, y desaparición de las lesiones dermatológicas; con lo que concluye adecuada respuesta al tratamiento.

Durante el internamiento se realizaron laboratorios de control en donde persistió FAN 2.3 Anti DNA $47 \mathrm{UI} / \mathrm{ml}$, C4 $11 \mathrm{mg} / \mathrm{dl}$, y una VES que fue descendiendo paulatinamente $(108 \mathrm{~mm} / \mathrm{h}$, $90 \mathrm{~mm} / \mathrm{h}, 24 \mathrm{~mm} / \mathrm{h})$.

Posterior a su egreso se inició descenso esteroidal y actualmente su tratamiento es con prednisona 5 mg/día VO y plaquinol 400 mg/día VO.

Actualmente lleva control en el Servicio de Reumatología con el diagnostico de Lupus eritematoso sistémico con manifestación inicial de una Enfermedad de Kikuchi-Fujimoto.

\section{DISCUSION}

La Linfangitis Histiocitica Necrotizante o Enfermedad de Kikuchi-Fujimoto (EKF) fue descrita por el Japones Masahiro Kikuchi en 1972 (1-2-3-4-14), quien describió una patología caracteriza por la aparición de fiebre y adenopatías preferentemente cervicales que afecta predominantemente a mujeres jóvenes ${ }^{(2-3-4)}$. La incidencia al día de hoy es desconocida ${ }^{(15)}$.

Es de etiología desconocida, aunque está cada vez más se está relacionando a procesos virales como posibles desencadenantes ${ }^{(2-5-14)}$. Se asocia a diferentes enfermedades del tejido conectivo, como enfermedad de Still, el síndrome de Sjögren, la polimiositis, la artritis reumatoide $y$ a LES principalmente ${ }^{(3-7)}$.

Clínicamente los pacientes desarrollan adenopatías cervicales y/o supraclaviculares que clásicamente son menores a $3 \mathrm{~cm}$ de consistencia firme y dolorosas a la palpación, fiebre, mialgias, pérdida de peso, náuseas, vómitos y lesiones cutáneas (2-4-8-15). Es importante siempre realizar diagnóstico diferencial con enfermedades infecciosas como toxoplasmosis, mononucleosis infecciosa, tuberculosis, parvovirus 19, Epstein-Bar entre otras; así como con linfomas (2-4-6-14-15).

En los análisis de laboratorio hay presencia de leucopenia, neutropenia y linfopenia, así como 
trombocitopenia, aumento de los reactantes de fase aguda, principalmente la Velocidad de eritrosedimentación (VES) ${ }^{(6-9-14)}$.

Histologicamente se presencian focos paracorticales de necrosis con abundantes restos nucleares cariorréxicos e infiltrados de histiocitos, con ausencia de neutrófilos ${ }^{(1-2-3-8-9)}$. Sin embargo la EKF es muy similar a la linfadenitis lúpica, se diferencian únicamente por la presencia de depósitos de $\mathrm{ADN}$ en la pared de los vasos o zonas de vasculitis fuera del área de necrosis que clásicamente nos sugiere presencia de linfadenitis lúpica y no $\mathrm{EKF}^{(10-14)}$.

La enfermedad de Kikuchi-Fujimoto es una entidad benigna. Generalmente es de resolución espontánea, posterior a un período de tiempo que va de semanas a pocos meses; por lo que generalmente los pacientes reciben tratamiento sintomático con antiinflamatorios no esteroideos (9-15-16). La recurrencia de esta entidad es mínima (16). Cuando la entidad se asocia a enfermedad inmunológicas como el Lupus Eritematoso Sistémico se agregan corticoides e inmunosupresores ${ }^{(4-9-15-16)}$.

\section{CONCLUSIONES}

En el caso de nuestra paciente el diagnostico se hizo concomitantemente, ya que durante su internamiento se le realizó tanto la biopsia de la adenopatía que fue concluyente para la EKF como los estudios inmunológicos que fueron compatible con LES; cabe destacar que si se descartaron patologías infecciosas, hematológicas y linfoproliferativas dado que el motivo de ingreso fue síndrome febril.

Concluimos que si bien es cierto, la EKF es una patología poco común en nuestro medio debemos sospecharla en pacientes con cuadros clínicos similares y vigilar su posible asociación con LES.

\section{BIBLIOGRAFÍA}

1. C. Diez-Morrondo et al / Reumatol Clin. 2012;8(2):153-155 Inicio de lupus eritematoso sistémico como enfermedad de Kikuchi-Fujimoto. Kikuchi Fujimoto's disease appearing as systemic lupus erythematosus.

2. Kucukardali Y, Solmazgul E, Kunter E, Oncul O, Yildirim S, Kaplan M. KikuchiFujimoto Disease: analysis of 244 cases. Clin Rheumatol. 2007;26:50-54.

3. Toledano Mũnoz A, García de Casasola G, Argüelles Pintos M, De los Santos Granados G. Enfermedad de KikuchiFujimoto: descripción de dos casos. Acta Otorrinolaringolaringológica Espa nola. 2006;57:152-154.

4. Paradela S, Lorenzo J, Martínez-Gómez W, Yebra-Pimentel T, Valbuena L, Fonseca E. Interface dermatitis in skin lesions of Kikuchi-Fujimoto's disease: a histopathological marker of evolution into systemic lupus erythematosus. 2008;17:1127-1135.

5. Lopez C, Oliver M, Olavarria R, Sarabia MA, Chopite M. Kikuchi-Fujimoto necrotizing lymphadenitis associated with cutaneous lupus erythematosus: a case report. Am J Dermatopathol. 2000;22:328-333.

6. Cramer J, Schmiedel S, Alegre NG, Schäfer H, Burchard GD, Merz H. Necrotizing

7. lymphadenitis: Kikuchi-Fujimoto disease alias lupus lymphadenitis? Lupus.2010;19:89-92.

8. Gómez García A, Martínez Hurtado E, Ruiz Ribera I. Enfermedad de KikuchiFujimoto asociada a infección por virus de la parotiditis. A propósito de 1 caso. An Med Interna. 2004;21:135-137.

9. Goldblatt F, Andrews J, Russell A, Isenberg D. Association of KikuchiFujimoto's disease with SLE. Rheumatology (Oxford). 2008;47:553554.

10. Gordon JK, Magro C, Lu T, Schneider R, Chiu A, Furman RR, et al. Overlap between systemic lupus erythematosus and Kikuchi Fujimoto disease: a clinical pathology conference held by the Department of Rheumatology at Hospital for Special Surgery. HSS J. 2009;5:169-177.

11. Casado E, Holgado S, Bielsa I, Rovira C, Llatjós R, Urrutia A, et al. Linfadenitis necrosante de Kikuchi-Fujimoto y su asociación con el lupus eritematoso 
sistémico: descripción de 4 nuevos casos. Rev Esp Reumatol. 2002;29:416-9.

12. Santana A, Lessa B, Galrão L, Lima I, Mittermayer S. Kikuchi-Fujimoto's disease associated with systemic lupus erythematosus: case report and review of the literature. Clin Rheumatol. 2005;24:60-3.

13. Quintás-Cardama A, Fraga M, Cozzi SN, Caparrini A, Maceiras F, Forteza J. Fatal

14. Kikuchi-Fujimoto disease: the lupus connection. Ann Hematol. 2003;82:1868.

15. Kampitak T. Fatal Kikuchi-Fujimoto disease associated with SLE and hemophagocytic

16. syndrome: a case report. Clin Rheumatol. 2008;27:1073-5.

17. Sopeña B, Rivera A, Vázquez-Triñanes C, Fluiters E, González-Carreró J, et al Autoimmune Manifestations of Kikuchi Disease. Elsevier Inc. Semin Arthritis Rheum 2012; 41:900-906

18. Calvo Romero JM. Enfermedad de KiKuchi-Fujimoto (Linfadenitis Necrotizante Histiocitaria). Rev Clin Esp 2002;202(2):94-5

19. Tsang WYW, Chan JKC, Ng CS. Kikuchi's lymphadenitis. A morfologic analysis of 75 cases with special reference to unusual features. Am J Surg Pathol 1994;18:219-31. 2. Thongsuksai P, Kayasut

\section{CONFLICTO DE INTERÉS Y/O AGRADE- CIMIENTOS}

Los autores declaran que no existió ningún conflicto de interés en el presente reporte. 